\title{
Design of Intelligent Detection Robot for Land and Air
}

\author{
Yang Ruiqing $^{1,2, a}$, Wei Hongmei ${ }^{1,2, b}$, Ji Zhiyong ${ }^{1, c}$ \\ ${ }^{1}$ School of Mechanical Engineering, Shandong Huayu University of Technology, Dezhou 253034, China \\ ${ }^{2}$ Dezhou Municipal Key Laboratory of Industrial Robot Control, Dezhou 253034, China

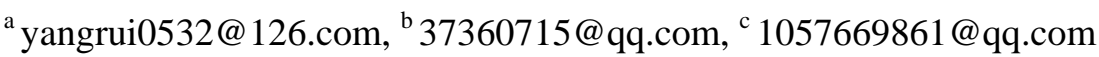

Keywords: intelligent detection robot, mechanical device, control system

\begin{abstract}
An intelligent detection robot that can realize air flight and land accesses is studied in this paper, mechanical structure and control system of the robot is designed. The robot is a new type of detection device, which consists of two parts: unmanned aerial vehicle and crawler. And these two parts are effectively combined. When the land is flat or the aircraft is limited, the rotor is contracted and the robot becomes car shaped. It can run smoothly on land like an all-terrain vehicle, with certain cross-country ability. When the terrain of the survey area is not smooth enough, the rotor opens and penetrates through the area in the form of an aircraft.
\end{abstract}

\section{Introduction}

The land and air intelligent detection robot is a multi-purpose detection locomotive that can replace traditional detection tools and carry out land and air accessibility[1-3]. The intelligent detection robot can be widely used in the fields of energy exploration, disaster relief, military reconnaissance, land and resources survey, land surveying, meteorological exploration, traffic supervision, industrial production, logistics transportation, personal consumption. Especially in complex and harsh environments, it can play an important role instead of manual operation. In the area of disaster relief, the existence of vital signs can be detected by manual control, and the device is transmitted to the command center through this device at the first time. The device has a powerful and reliable function with great practical significance. Moreover, it can achieve alternate cycling between land and air.

\section{Working Principle and Structure}

\subsection{Working Principle}

The control system is divided into automatic and manual control. In automatic control, the controller detects the specified area and records the data and route according to the prior input instructions. When the front ground is encountered, the rotor is expanded to continue the detection in the form of the aircraft. When encountering the ground ahead, the rotor can be recovered and detected in the form of vehicle detection. In the process of detection, the collected data in real time return back to the terminal, at the end of the mission, the controller automatically sends out instructions, makes unmanned aviation detection of robot according to the records of the work path automatically return, or by the operator planning new routes quickly. In manual control, instructions are sent to the controller by remote control or mobile ground station in the data terminal, controlling the detection robot to complete various work tasks. When the sensor on the robot detects that the front ground is unable to pass, it returns to the control terminal an inability to pass information, so that the person can do it accurately at the control terminal. The control schematic diagram is shown in Figure 1, and the control circuits diagram is shown in Figure 2. 


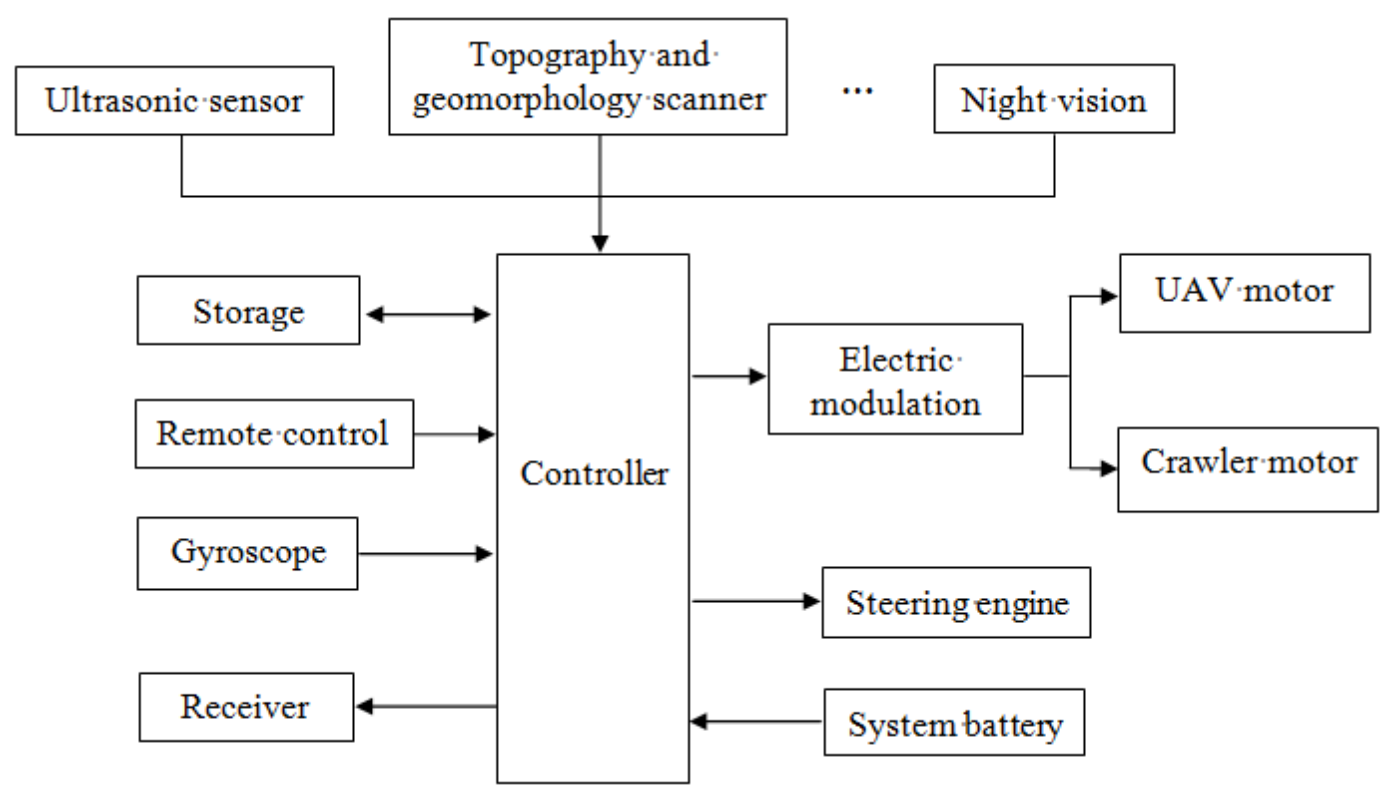

Figure 1 The control schematic diagram

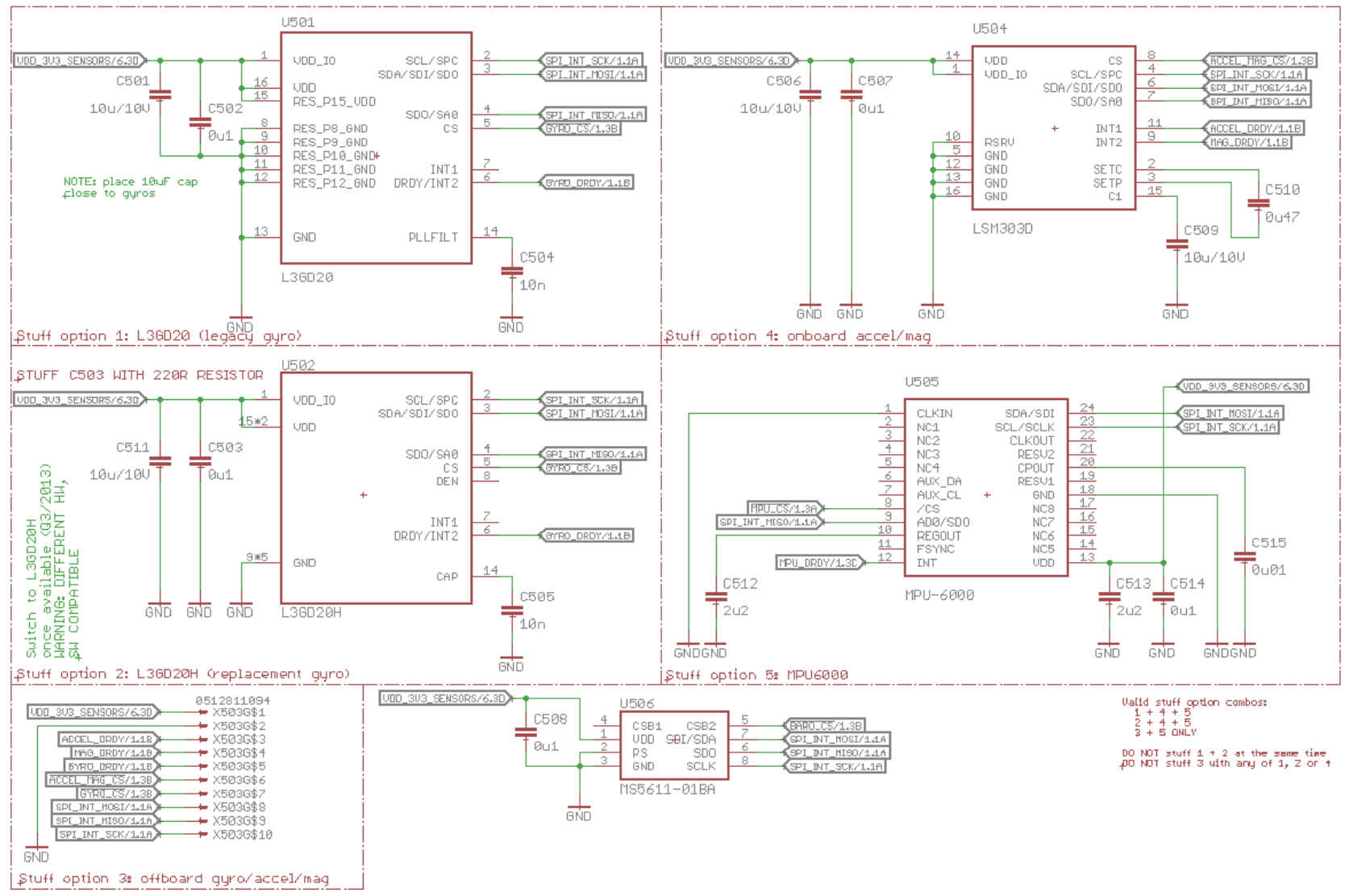

Figure 2 The control circuits diagram

\subsection{Design of Structure}

This robot mainly consists of three parts: the folding wing part, the chassis part and the function module part. The folding wing part mainly includes the main body of the wing and the transmission part of the rotor. The rotor is mainly driven by gears powered by two steering engines. The center of the driving wheel has a countersunk hole connected to the shaft of the steering gear. When the steering wheel rotates at a certain angle, it drives the gear to rotate and make the rotor rotate. By changing steering engine, the rotor can achieve contraction and expansion effect. The chassis part adopts a crawler system which can provide the driving force. The transmission chain analysis and 
modern simulation technology are applied to the design of the motion mechanism. The transmission ratio, rated power, maximum torque and other parameters of the machine power system are demonstrated and calculated. The main components of the motion mechanism are analyzed by finite element analysis. Component parameters enable the power system to provide maximum power.

The detection robot is installed the data transmission module, the graph transmission module, the GPS system and the Intelligent Cloud Computing large data processing system. It can detect the resource transmission control center in real time, and analyze the data in real time, greatly improving the efficiency. At the same time, the robot is installed a high power ultrasonic obstacle avoidance system. In the process of flying, it sends out the ultrasonic detection in real time. When the obstacle is detected in the front, the signal is sent to the remote terminal to request the obstacle avoidance or to avoid the obstacle in the automatic driving [4][5]. This greatly improves the ability of the detection robot to face the complex terrain.

When the remote control signal of the unmanned detection robot is disconnected, and the GPS signal is good, which can use its GPS autonomous navigation function to plan the return route, and guide the robot to return to the return point according to the planned route. The autonomous navigation is based on the information of the robot longitude, latitude and time provided by the receiver of the airborne global positioning system [6]. It provides feedback data for the planned route information, and combines the other parameters as the control parameters. It mainly controls the elevator and the aileron rudder of the detection robot, thus changing its flight attitude. It enables the robot to fly independently according to the planned route and return to the return point.

The intelligent detection robot can also realize one key automatic hovering, which is fixed at the preset height position and horizontal position, and the height position is measured by the barometer. The coordinates of the horizontal position are determined by the GPS module. When the GPS signal is not good, the detection robot can also hover with the "attitude mode", which relies on the inertial measurement unit within the controller, that is, a group of gyroscopes and accelerometers to identify their own flight state and relative displacement, and by hand, it can reach the predetermined position in hover. The device structure is shown in Figure 3.

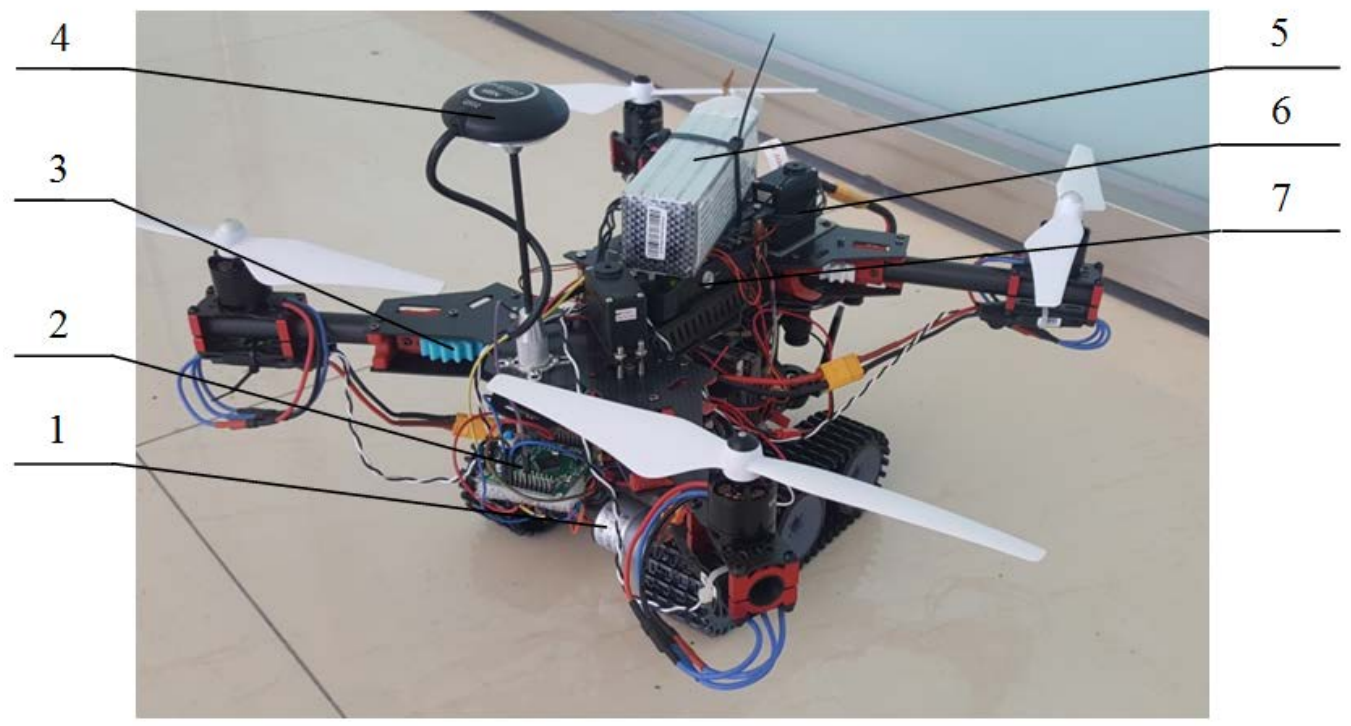

1. Chassis parts 2. Data transmission and graph transmission module 3. Folded wing,4. GPS, 5. Battery 6. Steering engine 7. Controller

Figure 3 The robot structure diagram

\section{Performance Parameters}

The following optimum parameters are obtained by a large number of experiments.

1) Takeoff weight: $4.5 \mathrm{~kg}$.

2) Maximum allowable speed: $30 \mathrm{~km} / \mathrm{h}$. 
3) Maximum climbing rate: $0.5 \mathrm{~m} / \mathrm{s}$.

4) Hover height: 51m.

5) Voyage: $30 \mathrm{~km}$.

6) Endurance time: $27 \mathrm{~min}$ (5000mAh 30C $4 \mathrm{~S}$ battery test).

7) Maximum wind resistance: level 4.

8) Maximum land speed: $20 \mathrm{~km} / \mathrm{h}$.

\section{Conclusions}

This paper develops a robot detection device suitable for both land and air. The performance parameters are obtained through experiments, and the stability of the system is proved. It can be widely used in the disaster relief, land surveying and so on. It can adapt to the requirements of complex terrain and air detection, and overcome the limitations of the independent work of the UAV and the detection vehicle.

\section{References}

[1] Mei L. K. (2013) Design and Implementation of Four Rotor Aircraft System Based on Embedded Technology. Beijing University of Posts and Telecommunications.

[2] Xie L., Han W. B. (2015) Design and Research of Four Rotor UAV Flight Control System. Optoelectronic Technology Application, 1,48-53.

[3] Long W. G., Wang A.L. (2008) Design and Simulation of Portable Four Wheel Track Robot. Machine Tool and Hydraulic Pressure.

[4] Chen M.Y. (2013) Intelligent Car Design Based on Image Recognition. Journal of Chongqing University of Technology, 248-251.

[5] Xu Y. (2013) Robot Positioning and Obstacle Avoidance Based on Ultrasonic Distance Measurement. Qilu University of Technology.

[6] Wang M., Guo B.X. (2000) Research on GPS Location and Road Matching Method in Vehicle GPS Navigation System. Journal of Wuhan University of Surveying and Technology. 